\title{
Supercontinuum generation in highly birefringent dual-mode fiber
}

\author{
Michalina Jozwik, ${ }^{* 1}$ Zbigniew Holdynski, ${ }^{1}$ Marek Napierala, ${ }^{1}$ Dawid Budnicki, ${ }^{1}$ Anna Ziołowicz, ${ }^{1}$ Pawel Mergo, ${ }^{2}$ \\ and Tomasz Nasilowski ${ }^{1}$ \\ ${ }^{1}$ InPhoTech Ltd., Slominskiego 17/31, 00-195 Warsaw, Poland, \\ ${ }^{2}$ Laboratory of Optical Fiber Technology, Maria Curie-Skłodowska University, Pl. Sklodowskiej 3, 20-031 Lublin, \\ Poland
}

Received March 10, 2016; accepted May 16, 2016; published June 30, 2016

\begin{abstract}
We present an experimental analysis of supercontinuum generation obtained for highly birefringent and highly nonlinear dualmode fiber. A specific difference between the zero dispersion wavelength of fundamental mode and higher order mode in such fiber offers the possibility of normal and anomalous dispersion regime pumping, depending on the mode which is excited. The nonlinear effects responsible for the generation of a broadband spectrum of light are analyzed when the fiber is pumped by a pulse pump source operating at a wavelength of $1064 \mathrm{~nm}$.
\end{abstract}

Supercontinuum (SC) generation is mostly achieved in nonlinear photonic crystal fibers with proper fiber geometry design [1]. When the dispersion characteristics of the fiber are well optimized, the pump source providing light with a high power density causes nonlinear effects responsible for broadening the spectrum of light. There are different nonlinear effects responsible for supercontinuum generation when different pumping regimes are applied, e.g. Stimulated Raman Scattering (SRS) and Four Wave Mixing (FWM) [2]. By proper fiber design, it is possible to change the dispersion characteristics of the fiber, in particular the zero dispersion wavelength (ZDW). The location of this wavelength has a strong influence on the mechanisms that cause supercontinuum generation [3]. The possibility of tailoring the dispersive properties in photonic crystal fibers is almost unlimited. Dispersion characteristics (and in consequence the ZDW position) can be modified especially in two ways - by the use of an appropriate material (e.g. silica glass or soft glass) or by varying the geometry of the fiber structure (in particular air-hole diameter and lattice constant). Thus it is possible to design the microstructured optical fiber with a specified dispersion, which is optimized for generation of nonlinear effects [4]. A slight change of structural parameters may affect the shape of the spectrum.

The efficiency of SC generation in a fiber is dependent on the difference between the pump source wavelength and ZDW of the fiber as well as on pump source parameters (e.g. power, pulse duration) and nonlinearity of the fiber [5]. Depending on the relation between ZDW and pump wavelength normal dispersion regime [6-7] or anomalous dispersion regime [8-9] pumping is realized and specific nonlinear effects are observed. In the case of the presented fiber, the proper relation between $\mathrm{ZDW}$ and pump wavelength allows the achievement of specific nonlinear effects responsible for supercontinuum generation.

The presented dual-mode fiber structure is comprised of air-holes of different sizes forming the geometrical layout of three parallel layers in the center of the optical fiber, as presented in Fig. 1. The core is $7 \mathrm{~mol} \%$ germanium doped and its diameter is $2.65 \mu \mathrm{m}$ and the fiber outer diameter is $125 \mu \mathrm{m}$.

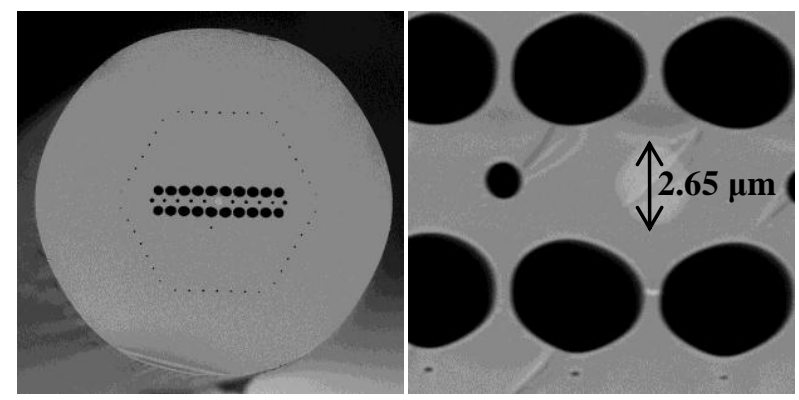

Fig.1. The structure of presented highly birefringent dual-mode fiber and zoom of its central area.

In the case of the presented dual-mode fiber, fundamental and second order modes are guided. Electric field distribution according to simulations of the FM and HOM is shown in Figs. 2-3.

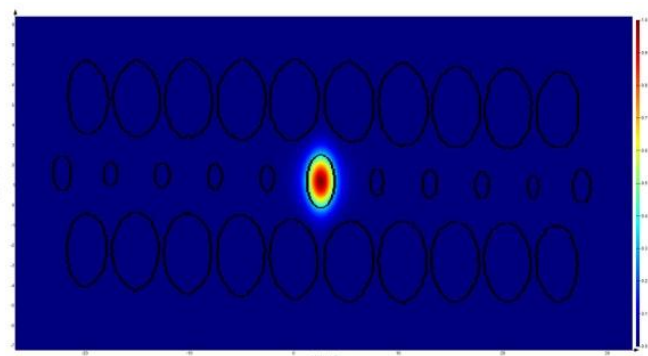

Fig. 2. Electric field distribution according to simulation of the fundamental mode.

\footnotetext{
*E-mail: mjozwik@inphotech.pl
} 


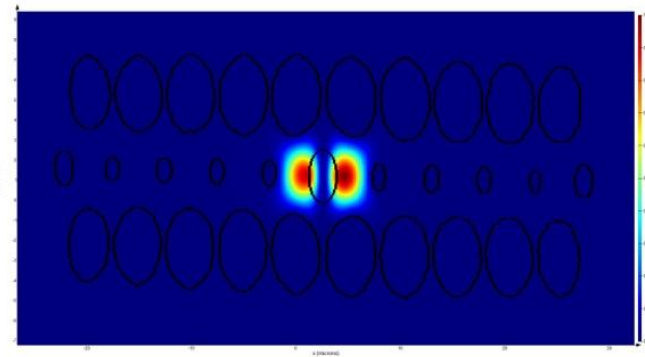

Fig. 3. Electric field distribution according to simulation of the second order mode.

The effective area of the fundamental mode and second order mode equals $8.06 \mu \mathrm{m}^{2}$ and $19.69 \mu \mathrm{m}^{2}$ respectively. The confinement losses are presented in Table 1.

Table 1. Simulated values of the effective refractive index $\left(n_{e f f}\right)$ and confinement losses of the first two propagated modes.

\begin{tabular}{|c|c|c|}
\hline \multirow{2}{*}{ Mode } & \multicolumn{2}{|c|}{ Simulation (at 1064nm) } \\
\cline { 2 - 3 } & $n_{\text {eff }}$ & $\begin{array}{c}\text { Confinement loss } \\
{[\mathrm{dB} / \mathrm{cm}]}\end{array}$ \\
\hline $\mathrm{FM}_{\mathrm{x}}$ & 1.450479 & $2.7547 \cdot 10^{-8}$ \\
\hline $\mathrm{FM}_{\mathrm{y}}$ & 1.450419 & $9.4352 \cdot 10^{-8}$ \\
\hline $\mathrm{HOM}_{\mathrm{x}}$ & 1.442072 & $3.9560 \cdot 10^{-8}$ \\
\hline $\mathrm{HOM}_{\mathrm{y}}$ & 1.441810 & $0.25105 \cdot 10^{-8}$ \\
\hline
\end{tabular}

The fiber was originally designed and manufactured for writing of Fibre Bragg Gratings (FBGs) and was used as a very sensitive strain sensor [10-12]. Here, we take advantage of dual-mode propagation which can be obtained by using the presented fiber to generate a broadband spectrum of light. Figure 4 presents the chromatic dispersion characteristics calculated for fundamental modes (FM) and higher order modes (HOM) for fiber (numerical simulations performed with the use of commercially available MODE Solutions software from Lumerical [13]).

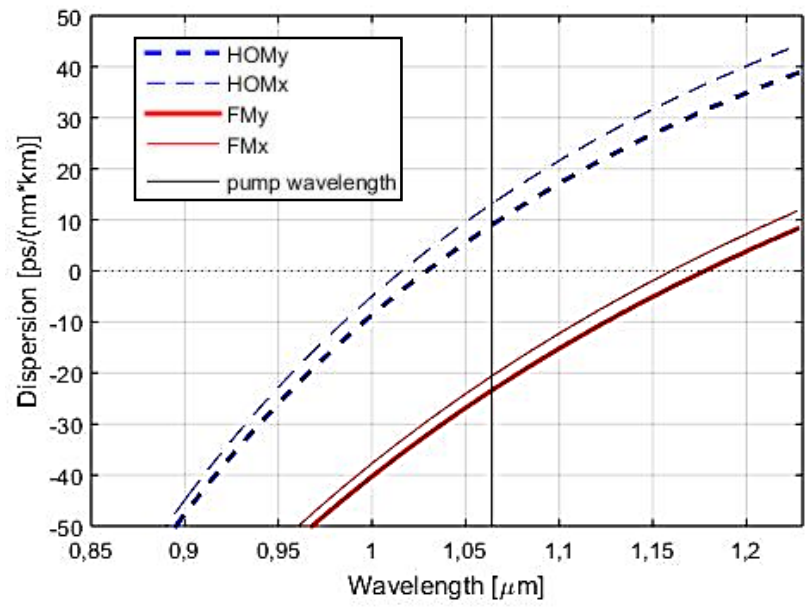

Fig. 4. Chromatic dispersion characteristics calculated for: fundamental modes (solid lines) and higher order modes (dashed lines) for the presented fiber.
The pump source operating at the wavelength of $1064 \mathrm{~nm}$ offers the possibility to obtain the condition where the fundamental mode $\left(\mathrm{ZDW}_{\mathrm{FMx}}=1159 \mathrm{~nm}\right.$, $\left.\mathrm{ZDW}_{\mathrm{FMy}}=1177 \mathrm{~nm}\right)$ is pumped in a normal dispersion regime, and higher order mode $\left(\mathrm{ZDW}_{\mathrm{HOMx}}=1015 \mathrm{~nm}\right.$, $\mathrm{ZDW}_{\mathrm{HOMy}}=1025 \mathrm{~nm}$ ) is pumped in an anomalous dispersion regime.

SC can be generated thanks to the nonlinear properties of the fiber. The pumping of the fiber in different dispersion regimes offers the possibility to receive various nonlinear effects. The fiber was pumped with the use of a pulse laser at a wavelength of $1064 \mathrm{~nm}$ with a pulse width of $0.7 \mathrm{~ns}$, average power of $140 \mathrm{~mW}$, peak power of $10 \mathrm{~kW}$ and pulse repetition frequency of $19 \mathrm{kHz}$. In Figure 3 the generated spectrum is presented.

The SC generation mechanism has an influence on the shape of generated spectra. The dominant nonlinear process in the early stage of SC generation depends on the relation between the pump wavelength and the $\mathrm{ZDW}$ position. Analysis of the evolution of an SC bandwidth near the pump wavelength gives detailed information about the type of dominant nonlinear phenomena. Figure 5 presents SC generation as a function of pumping power (pumping power was controlled by variable optical attenuator). Pump wavelength is indicated by vertical solid line and ZDW positions are indicated by dashed lines. The far field pattern of the signal at the output of fiber clearly shows the mode profile (HOM at short wavelength spectrum and fundamental mode at infrared regime). Different nonlinear effects are responsible for SC generation depending on the excited mode, since, due to different ZDWs of these modes, different dispersion regime pumping is obtained.

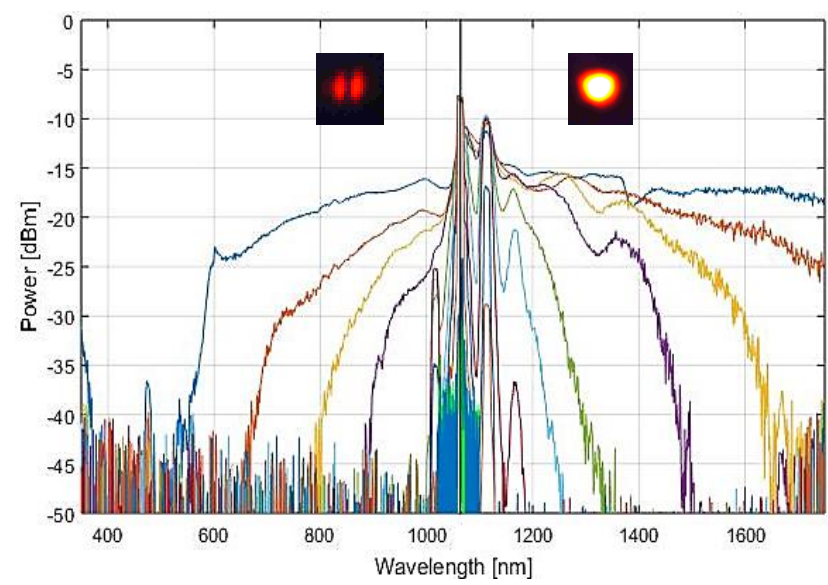

Fig. 5. SC generation in a function of pumping power. Vertical solid line indicates pump wavelength.

The broadband spectrum was generated in conditions when both modes (fundamental and HOM) were excited. This is due to a specific difference between their zero dispersion wavelength. SC generation in the fiber during pump power changes offers the possibility to observe 
both nonlinear mechanisms, which are characteristic of normal and anomalous dispersion regime pumping. In the case of the excitation of the fundamental mode, the condition in which the mode is pumped in a normal dispersion regime is obtained and SRS dominates. The process of SRS generation gives specific peak positions, as shown in Fig. 6. When HOM is excited, the condition in which the mode is pumped in an anomalous dispersion regime is obtained. In this case the cascaded FWM dominates. The initial process of SC generation produces an extremely narrow cascaded spectrum with characteristic FWM peaks, see Fig. 7.

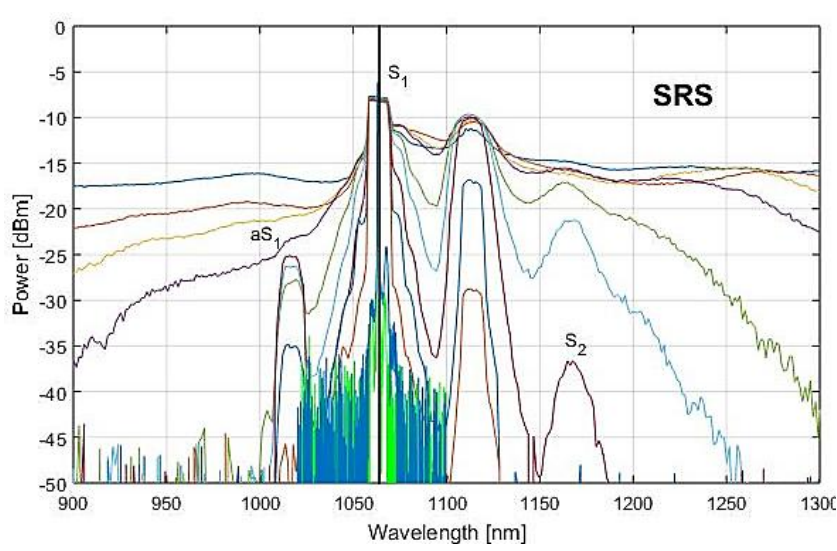

Fig. 6. Detail spectral evolution of the nonlinear effect - SRS.

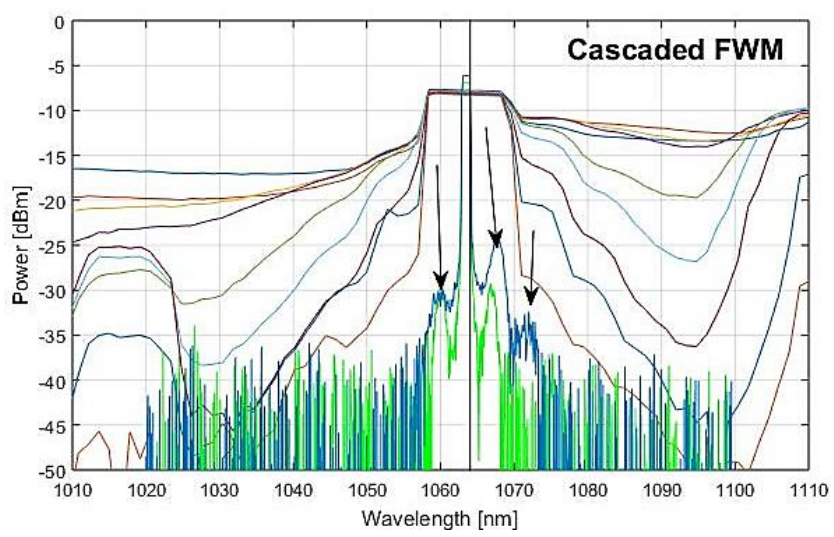

Fig. 7. Detail spectral evolution of the nonlinear effect - cascaded FWM.

In conclusion, supercontinuum generation in a highly birefringent dual-mode fiber pumped at a wavelength of $1064 \mathrm{~nm}$ in normal and anomalous dispersion regimes is achieved. As presented, dual-mode propagation can be used to generate a broadband spectrum of light (ranging from approx. $600 \mathrm{~nm}$ to more than $1650 \mathrm{~nm}$ ) in a specific way. Namely, different nonlinear effects responsible for supercontinuum generation and, in consequence, various broadening of light can be obtained using the same fiber, depending on the excited mode. The research connected with supercontinuum generation in such untypical fibers is important since it can be used in diagnostic or sensing systems. In addition, such a fiber allows an easy tuning of the generated spectrum by varying the excitation conditions (excitation of a different mode at the fiber input), which can be used in many applications. The next step of the research will be related with the exploiting the birefringence of the fiber and it will be focused on controlling polarization during supercontinuum generation.

The work described in this paper was partially supported by the Polish National Science Centre within the project 2013/09/N/ST7/04429.

\section{References}

[1] J. M. Dudley, G. Goëry, C. Stéphane, Rev. Modern Phys. 78(4), 1135 (2006).

[2] J.C. Knight, D.V. Skryabin, Opt. Expr. 15(23), 15365 (2007).

[3] Z. Holdynski et al., Opt. Expr. 21(6), 7107 (2013).

[4] M. Napierala et al., Proc. SPIE 91361, 91361M (2014).

[5] J.M. Dudley, J.R. Taylor, Supercontinuum generation in optical fibers (Cambridge University Press 2010).

[6] P. Falk, M.H. Frosz, O. Bang, Opt. Expr. 13(19), 7535 (2005).

[7] A. Hartung, A.M. Heidt, H. Bartelt, Opt. Expr. 19, 7742 (2011).

[8] B. Jinendra, K. Ranka, R.S. Windeler, A.J. Stentz, Opt. Lett. 25, 796 (2000).

[9] J.C. Knight et al., IEEE Photon. Technol. Lett. 12(7), 807 (2000).

[10] T. Tenderenda et al., Proc. SPIE 84260, 84260D (2012).

[11] T. Tenderenda et al., Opt. Expr. 20, 26996 (2012).

[12] K. Stepien et al., Opt. Lett. 39, 3571 (2014).

[13] http://www.lumerical.com/tcad-products/mode/ 